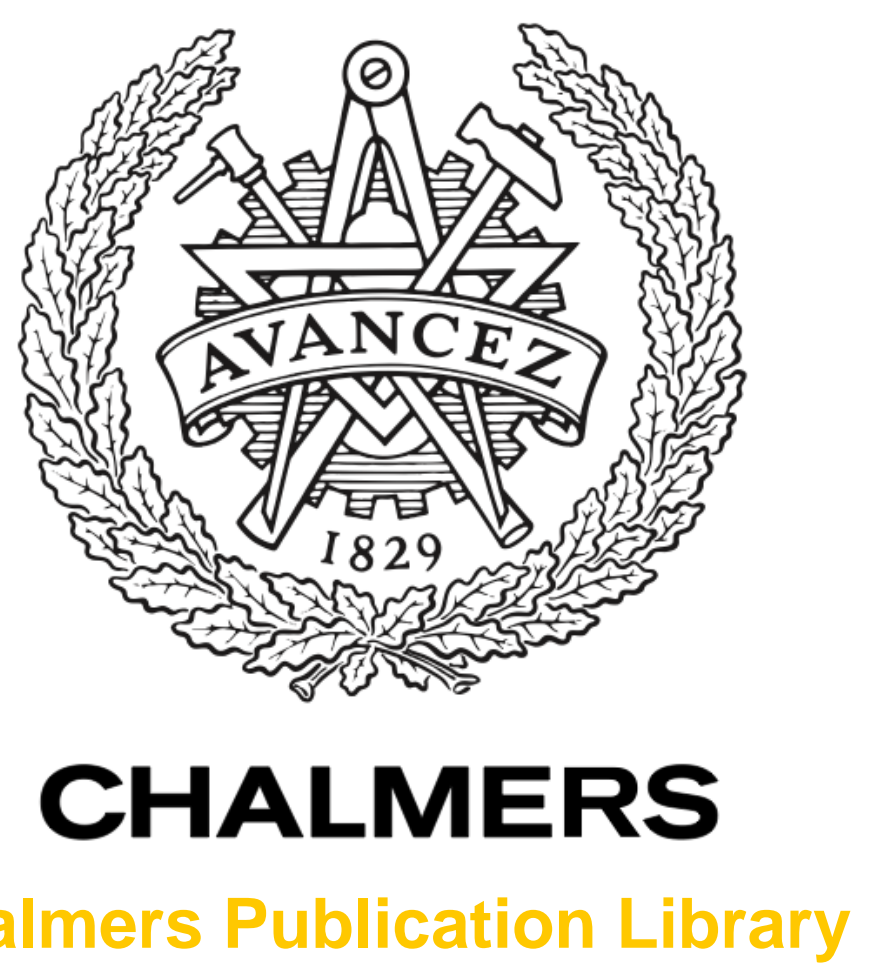

\title{
In-depth Analysis and Evaluation of Self-Organizing TDMA
}

This document has been downloaded from Chalmers Publication Library (CPL). It is the author's version of a work that was accepted for publication in:

\section{IEEE Vehicular Networking Conference (VNC)}

Citation for the published paper:

Gaugel, T. ; Mittag, J. ; Hartenstein, H. (2013) "In-depth Analysis and Evaluation of SelfOrganizing TDMA". 2013 IEEE Vehicular Networking Conference (VNC)

Downloaded from: http://publications.lib.chalmers.se/publication/191734

Notice: Changes introduced as a result of publishing processes such as copy-editing and formatting may not be reflected in this document. For a definitive version of this work, please refer to the published source. Please note that access to the published version might require a subscription. 


\title{
In-depth Analysis and Evaluation of Self-Organizing TDMA
}

\author{
Tristan Gaugel* ${ }^{*}$ Jens Mittag* ${ }^{*}$ Hannes Hartenstein*, Stylianos Papanastasiou ${ }^{\dagger}$, Erik G. Ström ${ }^{\ddagger}$ \\ \{tristan.gaugel, jens.mittag, hannes.hartenstein\}@kit.edu, stylianos.papanastasiou@ntu.ac.uk, erik.strom@chalmers.se \\ *Institute of Telematics \& Steinbuch Centre for Computing, Karlsruhe Institute of Technology (KIT) \\ ${ }^{\dagger}$ Dept. of Computing Science, Nottingham Trent University \\ $\ddagger$ Dept. of Signals and Systems, Chalmers University of Technology
}

\begin{abstract}
Recent studies suggest that Self-organizing TimeDivision Multiple Access (STDMA) might be a better medium access strategy in inter-vehicle communication networks than Carrier Sense Multiple Access (CSMA), especially when considering safety focused applications. Although it is necessary to completely understand a protocol and the effect of its 'turning knobs' on performance before adoption, STDMA has not yet been subjected to such rigorous treatment in the literature. In order to address this shortcoming we perform and present an in-depth analysis and evaluation of STDMA's fundamental principles. In particular, we contribute a detailed and complete description of the STDMA protocol, followed by the analysis and evaluation of two key questions: How can packet collisions occur in STDMA and whether packet collisions are 'contagious'. We further perform a fair comparison with CSMA on the basis of which we provide recommendations on the configuration of STDMA. Our results show that STDMA coordinates multiple access effectively - even in highly congested situations - as long as all transmitted packets are decoded successfully. When non-decodable (but still carrier-sensible) transmissions are present, STDMA effectiveness drops below that achieved by CSMA due to the lack of control information. To ensure reproducibility and encourage further inquiry we release the STDMA implementation used in this paper to the wireless networks research community.
\end{abstract}

\section{INTRODUCTION}

The potential of communicating vehicles which periodically exchange status messages to establish a mutual awareness and thus to cooperatively improve the safety level on the road has been placed in sharp research focus since the early 1990's. The success and significant evolution of the Carrier Sense Multiple Access (CSMA) based IEEE 802.11 standard family has furthermore prompted standardization bodies to adopt it, through the $802.11 \mathrm{p}$ amendment, as a basis for a first generation wireless inter-vehicle communications framework.

Recently, an alternative to CSMA is being investigated by several researchers: Self-organizing Time-Division Multiplexing (STDMA). While a station performs a random access to the wireless channel when using CSMA, STDMA, on the contrary, implements a reservation based scheme, which renders STDMA quite attractive for periodic data traffic. Furthermore, STDMA guarantees that each station gets a chance to access the channel and, according to recently published studies [8], [1], the protocol is claimed to coordinate multiple access better than CSMA. Hence, first efforts have been made to standardize
STDMA as an option in a second generation wireless intervehicle communications framework [3].

Before adopting a new coordination scheme and replacing CSMA with STDMA, it is essential to understand the alternative sufficiently well. While CSMA in general - and IEEE 802.11p for vehicular environments in particular - has been studied for more than a decade, studies on STDMA do not exist in significant numbers. Importantly, while the performance impact of the various configuration parameters of CSMA have been studied extensively and are well understood, the multitude of configuration parameters that exist in STDMA (as well as their performance impact) have not been adequately studied yet. It should be noted that both protocols have particular features in terms of handling varying packet lengths, ensuring predictability of transmission times and so on; however, not all such diverse aspects are considered here.

In this paper, we present a complete and detailed description of the STDMA protocol, followed by an in-depth, fundamental analysis and evaluation of the protocol elements that govern its function. We first analyze the protocol theoretically and then employ wireless network simulation to validate our findings in a static scenario. In particular, we consider two situations that the protocol has to handle, namely perfect and imperfect communication conditions and identify the causes that lead to reception failures in each. We are thus able to identify the limitations and weaknesses of STDMA that emerge only from the protocol itself, i.e. we can answer two fundamental questions: (1) how can slot allocation collisions occur in STDMA and (2) how do existing slot allocation collisions evolve over time? As no open or publicly available implementation of STDMA exists, we also contribute an implementation of STDMA to the well-known network simulator NS-3. ${ }^{1}$

The rest of this paper is structured as follows: Section II presents relevant work related to the paper topic. Section III provides a complete description of the STDMA protocol, followed by an analysis of STDMA in Section IV. Section V then validates and evaluates the results of the analysis by means of wireless network simulations. The paper concludes with a summary and outlook in Section VI.

\footnotetext{
${ }^{1}$ The implementation can be found at http://dsn.tm.kit.edu/ns3-stdma.php
} 


\section{RELATED WORK}

Extensive research on MAC protocols and specifically on coordinating the access on shared mediums has been ongoing for more than 40 years. Several different protocols have been proposed in the past, employing various approaches, on how to properly coordinate channel access. For a general introduction to MAC protocols for wireless networks, we refer the interested reader to a survey by Gummalla et. al [6]; in the following we solely focus on CSMA and STDMA.

CSMA deploys a 'listen before talk'-principle where nodes first listen to the channel and check whether a transmission is ongoing before transmitting themselves. In case the channel is sensed as busy, a random backoff is used before executing the next transmission attempt. CSMA has been deployed in multiple areas and has been extensively analyzed in the literature, e.g. under varying channel conditions [12] and the influence of protocol elements such as the contention window size, has been well understood [2]. Despite its random character, CSMA is capable of effectively coordinating multiple access on a wireless channel. For a more in-depth analysis and protocol description we refer to [12].

A distinct approach to CSMA, namely the self-organized TDMA (STDMA) was invented by Håkan Lans [11]. STDMA employs a reservation scheme where nodes communicate their next transmissions, resulting in fewer random elements compared to CSMA. The STDMA approach has been standardized and is used both in the Automatic Identification System (AIS) [7] as well as in VDL Mode 4 [4] to exchange positioning information between ships and aircraft, respectively. Available research on STDMA primarily focuses on the capacity and throughput of STDMA [5], particularly in maritime networks [10].

In recent work, the STDMA approach has been considered as a future option for VANETs, and initial results show promise. In particular, Sjöberg et. al evaluated the real-time properties of STDMA and found it superior to CSMA [8] and the hidden terminal problem in STDMA to be less severe, compared to CSMA [9]. Alonso et. al [1] investigated the stabilization time of STDMA and showed it to be superior (i.e. lower) compared to CSMA. In 2012, ETSI published a technical report [3], stating that STDMA exhibits slightly better performance, in terms of packet reception probability and channel access time, compared to CSMA. However, to the best of our knowledge, no in-depth analysis has been performed, to study the impact of protocol parameterizations on the performance of STDMA.

\section{STDMA PROTOCOL DESCRIPTION}

This section provides an overview of the STDMA protocol. As most other TDMA-based approaches, STDMA divides the time in so called frames that last for a certain duration, and those frames into equally sized transmission slots that accommodate a single packet transmission. The explanation given in this section is based on the latest version of the protocol specification in 2010 [7].

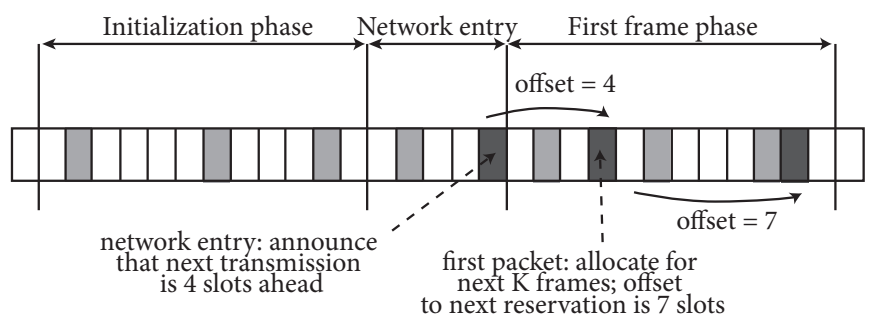

Slot detected as allocated by neighbors

Slot allocated locally

Fig. 1. Principles of STDMA: after startup, a station listens to the channel for a complete frame to determine the slot allocation status (initialization phase). In the following network entry phase the station performs a random access to announce its presence and its first slot reservation. The slot afterwards marks the beginning of the first frame phase during which further slot reservations and their duration are announced. Once every slot reservation within the first frame is transmitted, the continuous operation phase begins.

The structure of this section is as follows. First, basic assumptions and the fundamental protocol elements are explained in order to convey a basic understanding of the protocol. We then specify the random access strategy for transmitting the first packet, the slot reservation mechanism, and the slot re-reservation mechanism.

\section{A. Assumptions}

For clarity, but without loss of generalization, we assume

1) all stations generate packets at a fixed (report) rate $r$,

2) all packets adhere to a fixed maximum size $s$ (which translates to a fixed transmission duration),

3) internal clocks of all stations are synchronized, not in absolute terms (i.e. so that all stations share the same time) but such that all nodes know exactly when a new time slot starts and when it will end; note that synchronization of frame boundaries is not required.

In the following discussion the terms node and station are used interchangeably.

\section{B. Outline of Fundamental Principles}

The lifetime of a station in STDMA is divided into four different phases: initialization, network entry, first frame, and continuous operation. These ensure that each station first obtains an understanding of the slot allocation status, then announces its presence to the network, and afterwards performs the initial slot allocation for all transmissions to be made during one frame. Afterwards, the continuous operation phase is entered in which only slot re-allocations are carried out.

Using these terms, Figure 1 explains the fundamental principles that are applied in the network entry and the first frame phase. After having listened to the channel for one complete frame, the next $i$ slots are considered for random access. The number of slots is subject to configuration (e.g. [7] sets $i$ to 150 slots). The station then randomly selects an available slot out of these $i$ slots in order to

1) introduce its presence to the network,

2) and pre-announce the next slot it is going to use

Hence, before transmitting the network entry packet, the station already has to decide which slot it will use for its first 
reservation. As a result, neighboring stations that receive the network entry packet become aware of the presence of the station and the transmission slot it is going to use next.

When using this first pre-announced transmission slot (termed first packet in Figure 1), the station repeats the same process: it announces the next slot it is going to use, and in addition indicates how often it will re-use the current slot. In STDMA, the terms timeout and offset are used to refer to the duration of a reservation in frames and the difference in slots between two transmission slots. Each STDMA packet must contain a timeout value, an offset value, and the geographical position of the sender.

In contrast to previous descriptions of the STDMA protocol, we assume that the packet responsible for network entry does not belong to the first frame. Instead, we assume that the first frame starts right after network entry.

\section{Initialization Phase}

During the initialization phase, a station is only listening to the channel and monitoring the status of each single slot. Each slot can be in one of the following states (in order of decreasing priority):

1) Internally Allocated: a slot is considered to be internally allocated if the station has allocated this slot to itself. The state is maintained as long as the internal timeout value is greater than zero.

2) Externally Allocated: a slot is considered to be externally allocated if a transmission has been observed in the past that indicated that this slot is going to be used. Note that it is not required that this past transmission has been observed in the same slot. It is also possible that this external allocation has been communicated through the offset parameter in a different slot. The state externally allocated is maintained as long as the recorded timeout value is greater than zero.

3) Busy: a slot is considered to be busy if either (a) a packet has been detected in the same slot of the previous frame but the packet failed to be decoded successfully, or if (b) the average energy level at the receiver during the same slot of the previous frame was above the configured clear channel assessment (CCA) threshold ${ }^{2}$. The busy state is kept until the end of the next frame.

4) Free: a slot is considered to be free if all of the following conditions hold: (a) no observed transmission in the past has indicated that this slot is going to be used in the future; (b) no preamble was detected in this slot; (c) the average energy level at the receiver measured during the same slot of the previous frame was below the configured CCA threshold ${ }^{3}$.

After the station has listened to the channel for one frame, it should have a good understanding of the slot allocation status in the network and be able to derive sound allocation decisions

\footnotetext{
${ }^{2}$ The state busy is not explicitly modeled in [7] but has been added for clarification by the authors.

${ }^{3}$ The conditions (a) and (b) are not explicitly stated in [7] and have been added for clarification by the authors.
}

for the next frame(s). The monitoring activity is never stopped to keep the station updated over time. When updating the local slot allocation status table, each station uses the following policies: (1) the state observed in the previous frame is only overwritten if the new state is of higher priority; (2) when a slot is already marked as externally allocated upon reception of a packet, the maximum of the old and new timeout values is used; (3) a state transition from externally allocated to free is only applied if the reservation duration has expired, or if the slot has been detected to be free at least 3 consecutive times.

\section{Network Entry Phase}

The network entry phase is the time period starting directly after the initialization phase and ending when the packet that introduces the station's own presence has been broadcast. This packet is referred to as the network entry packet in the following. The selection of the time slot for this network entry packet is based on the random access time-division multiple access (RATDMA) protocol of [7] and is specified as follows:

1) Define a selection interval (SI) of potential transmission slots and let it cover the next $i$ transmission slots (e.g. $i=150)$

2) Establish a set of potential transmission slots by including all slots marked as free in this $\mathrm{SI}^{4}$.

3) At the beginning of each potential transmission slot, randomly decide whether to use this slot or not.

Essentially, the RATDMA protocol employs a p-persistence mechanism to decide in which of the potential transmission slots the network entry will be performed. The probability $p(k)$ for a transmission in a potential transmission slot $k$ is defined as

$$
\begin{aligned}
& p(0)=\frac{1}{n(0)} \\
& p(k)=p(k-1)+\frac{1-p(k-1)}{n(k)}, k>0
\end{aligned}
$$

where $n(k)$ denotes the number of slots remaining in the potential transmission set, beginning from slot $k$ to the last slot. Importantly, the number of free slots, denoted by $n(k)$, is updated (i.e. reduced) in case one of the potential transmission slots is detected as externally allocated. The exact definition is given in $\S 3.3 .4 .2$ of [7].

Since confusion exists in the literature it is worth re-iterating that the network entry packet is a one-time transmission in order to announce the first packet of the first frame, i.e. the network entry packet signals the offset to the next slot being used. A description of how this next slot is selected follows.

\section{E. First Frame Phase}

Once the station has announced its presence, the station enters the first frame phase. The objective of this phase is to announce and reserve additional slots in order to fulfill the configured report rate. Assuming that one frame comprises $N$ slots and the station is configured to transmit $r$ packets per frame, the allocation is performed step-by-step as follows:

\footnotetext{
${ }^{4}$ In this matter we diverge from [7], by not considering externally allocated slots for the network entry as long as at least one free slot within the $S I$ is available.
} 


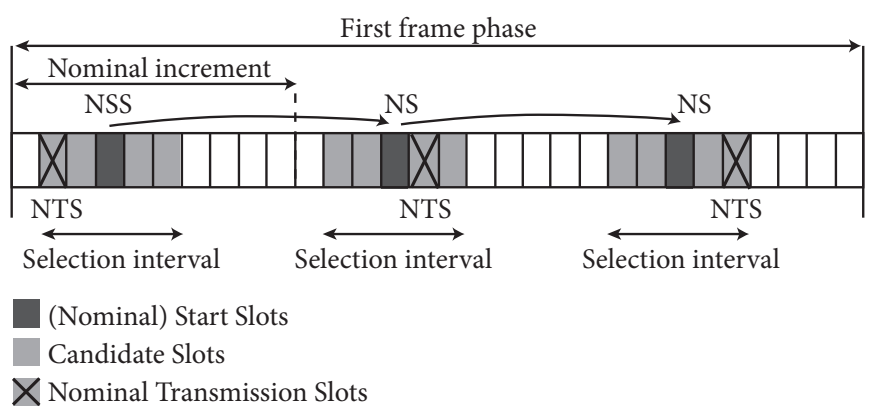

Fig. 2. First frame phase: one of the first nominal increment (NI) slots is randomly selected as nominal start slot (NSS).

1) Set the nominal increment (NI) value to $\lfloor N / r\rfloor$.

2) Randomly select a nominal start slot (NSS) out of the first NI slots.

3) Derive additional $r-1$ nominal slots (NS) by subsequently adding NI slots to NSS.

4) For the NSS and each NS:

a) Construct a selection interval (SI) by adding $\lfloor(N / 2 r) s\rfloor$ number of slots to the left and to the right, with $s$ being the SI ratio, e.g. $20 \%$.

b) Compile a set of candidate slots (see Section III-G for more details) within this SI

c) Randomly choose one of these candidate slots as nominal transmission slot (NTS)

When announcing the allocation of a selected slot, a random timeout value is drawn from statically defined minimum and maximum timeout limits. Hence, each allocated slot gets its own timeout value.

\section{F. Continuous Operation Phase}

After the first allocation within the first frame phase is performed, the station enters continuous operation. During this phase a station performs re-allocations whenever the internal timeout of a slot expires. The rules for re-allocation are the same as in the first frame phase, however, a station is allowed to stick to the current slot if no candidate slot is available. Furthermore, when re-reserving a slot, the semantics of the offset value changes. While it normally indicates the offset to the subsequent transmission, it then indicates the offset to the newly selected slot in the next frame.

\section{G. Candidate Slot Set Compilation}

The compilation of candidate sets is, as seen earlier, needed throughout slot reservation and re-reservation. According to §3.3.1.2 of [7] it has to adhere to the following set of rules:

- There should always be at minimum $\mathcal{C}$ candidate slots to choose from unless the number of candidate slots is otherwise restricted due to lack of position information (e.g. when there are too many busy slots in the selection interval).

- The candidate slots are primarily selected from free slots.

- If less than $\mathcal{C}$ slots are free, externally allocated slots are included in the candidate set.

- Externally allocated slots are added in order of decreasing distances to the current station.

\begin{tabular}{l|l}
\hline Parameter & Value \\
\hline Min. number of candidate slots & 4 slots \\
Frame duration & 1 sec \\
Min. reservation timeout & 3 frames \\
Max. reservation timeout & 7 frames \\
Selection interval ratio & 0.2 \\
Max. duration of network entry & 150 slots \\
Guard interval (per slot) & $6 \mu s$ \\
\hline \multicolumn{2}{c}{ TABLE I } \\
DEFAULT PROTOCOL CONFIGURATION ACCORDING TO [7]
\end{tabular}

- Externally allocated slots may only be added if the (local) station is not already intentionally reusing another slot of the same (remote) station.

- All candidate slots have the same probability of being chosen, regardless of their state.

- If there are still less than $\mathcal{C}$ candidate slots available the station selects one slot out of the reduced candidate set.

- If no candidate slots can be found, the station should not reserve a slot in the SI. This is unlikely to happen since this would only be the case if all slots are un-decodable.

With the rule set above, each station can select the slots to be used. The rule set aims to ensure that simultaneous transmissions are either avoided or spatially separated as much as possible. In the latter case, it is further the objective to reuse at most one slot of the same remote station.

\section{H. Parametrization}

The following protocol parameters can be tuned: minimum number of candidate slots, frame duration, minimum and maximum reservation timeout, selection interval size, maximum number of slots to consider for random access during network entry, and guard interval between two slots. Table I shows the values considered as default by [7]. The only changes made are a reduction of the frame duration to one second and the guard interval has been adjusted to protect against the maximum worst-case path delays in $5.9 \mathrm{GHz}$ radio channels.

\section{PRotocol Analysis}

We now analyze how packet (or slot allocation) collisions can occur in STDMA, i.e. which events lead to a situation in which two stations transmit at the same point in time, and how do such packet collisions then affect the coordination efficiency of the protocol.

To address the first question, we assume that all stations are within each other's communication range, that all packets that are received in the absence of interference can be decoded successfully, and that the number of slots per frame is greater than the number of slots required to satisfy each stations demand. Then, only the following three situations can lead to packet collisions:

1) Two (or more) stations startup at approximately the same time and randomly select the identical slot for network entry.

2) All random access slots of a station's network entry phase are marked as busy or externally allocated. In this case, the entering station will reuse the slot allocated by the station furthest away. 
3) The candidate slot set has been enriched to satisfy the minimum number of candidate slots requirement and an externally allocated slots was selected while performing a (re-)reservation.

The first situation can be dismissed as an unlucky occurrence and there can be no counter measure against it in traditional wireless networks, which are incapable of 'listenwhile-talk'. The second situation can easily occur if the maximum duration of the network entry phase is too short to find a gap of available slots in the frame.

The third situation can, on the one hand, occur if there are still free slots available within the SI, but too few to satisfy the configured number of required slots in the candidate set. In this case, even though free slots are available an externally allocated slot might be selected. This situation can easily be avoided by reducing the number of required candidate slots to one. On the other hand, if no free slots exist in the SI, the candidate set contains only externally allocated slots, forcing the station to (knowingly) re-use a slot. An immediate question is therefore: how easily can STDMA run into this last situation? Clearly, this situation is triggered when the channel is overloaded, but can also otherwise occur whenever the nominal (start) slots of all neighboring nodes are not uniformly distributed and the selection interval size is not large enough to avoid local congestion. As soon as the nominal (start) slots accumulate in certain areas of a frame the channel becomes overloaded in these areas and remains unused in the areas not covered by the selection intervals. One solution to mitigate this effect is to increase the selection interval size, which however has the drawback that packet inter-transmission times become less predictable.

It should be noted that the above reasons for packet collisions are of statistical nature. Hence, it is possible that these events do not occur at all over a given time period as their probability of occurrence is very low. Further, additional influences such as station mobility (which is not considered in this work due to space constraints) can also lead to slot allocation collisions. Yet, independent of the reason, the consequence is always the same: transmitted control information (slot offsets and reservation durations) are prone to be non-decodable by remote stations, due to drastically reduced SINR resulting from packet collisions in these slots. Eventually, this might prohibit these remote stations from obtaining a proper understanding of the slot allocation status or re-reservation decisions.

An additional important question to answer is how existing slot allocation collisions in particular, or the lack of control information in general, affect subsequent coordination decisions of STDMA. Specifically, it is worth investigating whether slot allocation collisions are contagious as the lack of control information might support the introduction of new allocation collisions in subsequent re-reservation processes, or whether the protocol is able to resolve the situation. To answer this question, we refer to the microscopic example in Figure 3(a) which illustrates the slot allocations of four stations using a transmission rate of $2 \mathrm{~Hz}$. As can be seen stations 1 and 2 are not involved in any allocation collision, however,

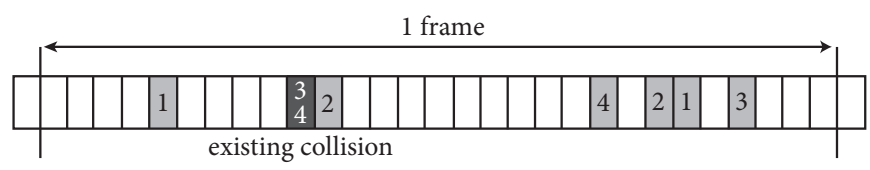

(a) Before re-reservation process

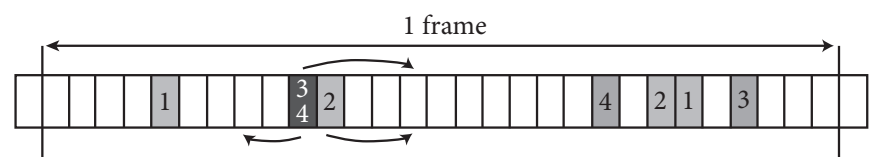

(b) Reservation decisions of stations 2, 3 and 4

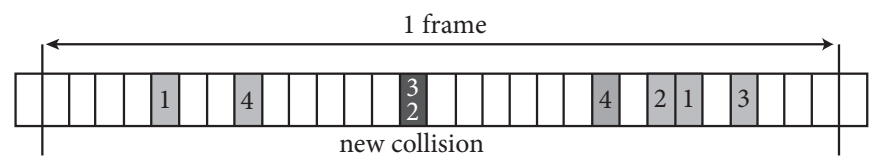

(c) After re-reservation process

Fig. 3. Illustration of what can happen during re-reservation of two stations that are already involved in a slot allocation collision: (a) allocation status before re-reservation; (b) decisions made by stations during re-reservation; (c) allocation status after re-reservation.

stations 3 and 4 use the same slot for their first transmission ${ }^{5}$. Consequently, station 1 and 2 will not be able to successfully decode the information in this slot, hence, they do not know for how long this slot will be used. Even more importantly, they will miss the notification to which slot station 3 and 4 switch once they perform a re-reservation. What can happen then is illustrated in Figure 3(b): assuming that both, station 3 and 4, as well as station 2 perform a re-reservation in the same frame, the probability arises that station 2 selects the same slot as station 3 and/or station 4 . As station 2 performs a re-reservation after these two stations (while not being able to decode their re-reservation notification), it is not aware of the newly selected slot(s) and may choose (with a certain probability) the same slot. Hence, if there is already a slot allocation collision existing, the probability exists that either the same, one or more than one new collisions are present after the re-reservation process. One of these outcomes is exemplarily illustrated above in Figure 3(c).

\section{Simulation-Based Evaluation}

In this chapter we present the results of a simulationbased performance evaluation of STDMA and compare the results against a CSMA-based medium access control implementation. We first introduce our evaluation methodology and performance metrics, and then describe the set of conducted experiments. Finally, we present and interpret the obtained results.

\section{A. Methodology}

We group our experiments into two sets, both of which represent fundamental situations that any distributed medium access control has to address: (1) perfect, and (2) imperfect decoding capabilities. In both connectivity scenarios, stations

\footnotetext{
${ }^{5}$ Without loss of generality and for the sake of simplicity we assume in this example that all stations use the same frame boundary.
} 


\begin{tabular}{l|l}
\hline Scenario and application layer \\
\hline Number of stations & 42,63 , and 84 stations \\
Packet size & $400 \mathrm{bytes}$ \\
Transmission rate & $10 \mathrm{~Hz}, 20 \mathrm{~Hz}$ \\
1 node/sec deterministic, \\
Startup behavior & 4 nodes/sec randomized \\
\hline CSMA based medium access control \\
\hline Slot time & $13 \mu s$ \\
Contention window size & 15 slots \\
\hline STDMA based medium access control \\
\hline Frame duration & 1 sec \\
Initialization phase duration & 1 frame +1 selection interval \\
Min. candidate slots & 1 slot, 4 slots \\
Reservation duration & 3 frames, 7 frames, $\mathcal{U}[3,7]$ frames \\
& endless reservation \\
Selection interval ratio & $20 \%, 40 \%, 60 \%, 80 \%, 100 \%$ \\
Network entry duration & 150 slots \\
Guard interval per slot & $6 \mu s$ \\
\hline
\end{tabular}

TABLE II

APPLICATION AND MEDIUM ACCESS CONTROL LAYER PARAMETERS USED.

are placed on a straight road segment and are not moving. The wireless channel is configured such that each station can sense each other's packet transmissions. Additionally, in the first connectivity scenario all stations can successfully decode each other's transmissions as long as there is no interference (i.e. an interfering transmission will prohibit the successful reception of reference and interfering transmission). In the second connectivity scenario, successful decoding of transmissions is only possible if the distance between sender and receiver is less or equal than approx. $60 \%$ of the carrier sensing range. This artificial limitation of the range over which packets can successfully be delivered, ensures an effective limitation of the degree up to which a common view on the reservation status can be established among stations. Note that it does not matter where this limitation comes from. For instance, one could also introduce fading channel effects in order to introduce decoding errors. However, one has to ensure that the load each station is exposed to, such as the amount of transmission (in Mbps) that can be sensed, does not change otherwise the results are not comparable.

In both connectivity scenarios we simulate several different numbers of vehicles: 42, 63 and 84. Together with a configuration of 400 byte data packets, a transmission rate of $10 \mathrm{~Hz}$ and $20 \mathrm{~Hz}$, and a physical layer data rate of $6 \mathrm{Mbps}$, the stations generate exactly $25 \%, 50 \%, 75 \%$ and $99 \%$ channel congestion ${ }^{6}$. The medium access control implementation is set once to CSMA and once to STDMA in each setup. When running an experiment with STDMA, we further vary the minimum number of candidate slots, the reservation duration and the selection interval ratio according to the values given in Table II. To study the influence of protocol startup, we simulate each scenario twice: once with all stations starting up one after the other (using a little bit more than one frame as separation which constitutes the best case for STDMA, since two nodes cannot select the identical slot for their network

\footnotetext{
${ }^{6}$ To clarify: when using a 6 Mbps data rate, $50 \%$ channel congestion refers to $3 \mathrm{Mbps}$ of transmissions that can be carrier sensed.
}

\begin{tabular}{l|l|r|r|r|r}
\hline \multirow{2}{*}{$S I$} & Min CS $(\mathcal{C})$ & \multicolumn{2}{|c|}{3 frame reservation } & \multicolumn{2}{|c}{ endless reservation } \\
& Min value & Max value & Min value & Max value \\
\hline $20 \%$ & 1 slot & $1.6 \%$ & $6.8 \%$ & $4.4 \%$ & $6.6 \%$ \\
& 4 slots & $8.3 \%$ & $11.5 \%$ & $9.1 \%$ & $10.9 \%$ \\
\hline $40 \%$ & 1 slot & $1.6 \%$ & $2.8 \%$ & $1.9 \%$ & $3.2 \%$ \\
& 4 slots & $13.1 \%$ & $13.8 \%$ & $4.6 \%$ & $5.5 \%$ \\
\hline $60 \%$ & 1 slot & $1.5 \%$ & $3.1 \%$ & $1.2 \%$ & $1.6 \%$ \\
& 4 slots & $12.1 \%$ & $12.7 \%$ & $2.8 \%$ & $2.9 \%$ \\
\hline $80 \%$ & 1 slot & $5.8 \%$ & $7.8 \%$ & $0.8 \%$ & $1.0 \%$ \\
& 4 slots & $12.9 \%$ & $13.1 \%$ & $2.0 \%$ & $2.4 \%$ \\
\hline $100 \%$ & 1 slot & $10.2 \%$ & $11.7 \%$ & $0.6 \%$ & $0.9 \%$ \\
& 4 slots & $14.1 \%$ & $14.3 \%$ & $1.5 \%$ & $1.8 \%$ \\
\hline
\end{tabular}

TABLE III

Minimum AND MAXIMUM PLI VALUES THAT WERE OBSERVED IN THE 84 STATION AND $20 \mathrm{HZ}$ SCENARIO, WHEN USING EITHER A 3 FRAME OR ENDLESS FRAME RESERVATION DURATION.

entry), and once with stations starting up randomly using a rate of 4 nodes/s.

Before we evaluate the protocol performance, we wait until all stations are started up, and then monitor each station for the next 170 seconds to track its perception and to collect all the data needed to quantify the performance metrics described below. To achieve statistically significant results, each setup is simulated 20 times with a different seed for the random number generator. To evaluate the performance, the following primary performance metric is used.

Definition 1 (Packet Level Incoordination, PLI): The packet level incoordination, as observed from the perspective of a node $r$ and one of its generated packets $p$, describes the probability that, at least, one node $s, s \neq r$, transmitted a packet $q$ during the transmission period of $p$.

We further collect information to construct packet transmission rate and slot occupation count distributions. The packet transmission rate is used to check whether all stations transmitted as many packets per second as generated on application layer. The slot occupation statistic indicates how many slots remain unused and how often more than two nodes allocate one slot.

\section{B. Results for perfect decoding capabilities}

1) Low to medium channel congestion: We start with summarizing the results for the setup with 42 stations using a fixed packet generation rate of $10 \mathrm{~Hz}$. Together they generate a load of $25 \%$, which is low enough to generate no packet-level incoordination at all, no matter how the STDMA protocol is configured. This performance is expected, as the network entry collision reasons listed in Section IV are either very unrealistic (identical slot for network entry) or have been avoided as the initialization phase has been extended in order to cope with the underlying issue. (Re-)reservation collisions just do not happen as the selection interval spans 34 slots in the worst case ( $S I$ of $20 \%$ ) and it is unlikely that the nominal start slots generate a local congestion that is dense enough to block all of them. Needless to say that $24.8 \%$ of the slots are used exactly by one station, and $75.2 \%$ not at all.

The situation changes if the 42 stations generate 20 packets/sec, increasing the offered load to $50 \%$, as the config- 
uration of STDMA does slightly matter now. Although the level of incoordination is still very low (ranging from $0.02 \%$ to $0.18 \%$ ), all configurations in which a selection interval size of $20 \%$ (which equals 17 slots) in conjunction with a minimum candidate set size of 4 is used exhibit incoordination. As investigations reveal, the (re-)reservation collision reason strikes in these setups, i.e. there would be slots available, but as the candidate set had to be enriched with externally allocated ones, there is a chance that an already externally allocated slot is selected. Apparently, these slot collisions could have been avoided. It also turns out that the problem is not related to the congestion level at all. If 84 stations generate 10 packets/sec, which equals to an offered load of $50 \%$ as well, the probability of incoordination reason number 3 drops to zero again as the selection interval size is increased to 34 slots due to the reduced packet generation rate.

2) High channel congestion: Incoordination becomes almost inevitable if a setup with 84 stations and a packet generation rate of $20 \mathrm{~Hz}$ is used. This combination yields a congestion level of approx. 99\% (1680 required slots versus 1694 available slots in total per frame). Table III lists the recorded PLI values over a wide range of protocol configurations: different selection interval sizes, one or four required candidate slots, and two different slot reservation durations. The influence of the network entry duration has been abstracted in this table due to space limitations, but is indicated through the minimum and maximum observed PLI value in each combination of the above settings.

The first immediate observation is the negative impact of the requirement of at least 4 candidate slots. As already pointed out before, this leads to incoordination that could have been avoided.

Second, the effect of an increased SI-size for a 3 frame reservation is contrary to endless reservation - increasing PLI with increased SI size for 3 frame reservation and decreasing PLI with increased SI size for endless reservation. This can be explained by the fact that with endless reservation there is only one initial slot reservation per nominal slot taking place. As discussed in the last section, this reservation can only be successful if there is, at least, one free slot available within the selection interval. Consequently, the probability of a successful initial slot reservation increases with a larger SI size. Importantly, even with an SI-size of $100 \%$ the resulting selection interval (with a transmission rate of $20 \mathrm{~Hz}$ ) results in only 84 slots. Thus, with a total of 14 free slots within a frame of 1694 slots and possibly non equally distributed nominal slots, this can still lead to collisions due to lack of free slots within the SI. With a reservation duration of only 3 frames the PLI tendency is the opposite. In contrast to endless reservation, there is not just one initial reservation, but in addition one re-reservation taking place per NS every three seconds. As discussed in the protocol analysis, slot allocation collisions can be rather contagious and lead to a lot of new collisions, comprising previously collision-free nodes. In our scenario, with a high load of $99 \%$ and frequent synchronized rereservations every 3 seconds exactly this effect is observable.

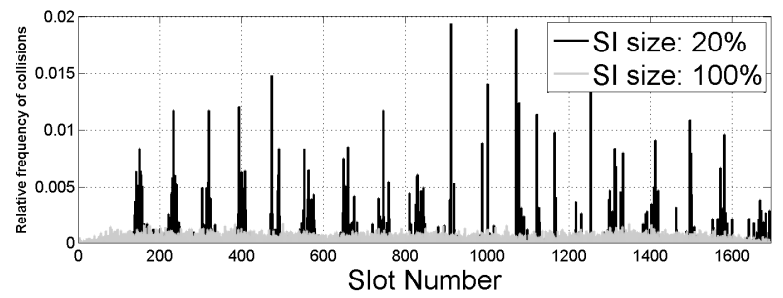

Fig. 4. Slots in which collisions occurred in the $99 \%$ load scenario with a 3 slot reservation duration and an SI of $20 \%$ and $100 \%$, respectively.

\begin{tabular}{l|c|c|c}
\hline \multirow{2}{*}{ \#nodes/rate } & CSMA & \multicolumn{2}{c}{ STDMA } \\
\hline $42 / 20 \mathrm{~Hz}(\approx 50 \%$ load $)$ & $1.07 \%$ & $0 \%$ & $0 \%$ \\
\hline $84 / 10 \mathrm{~Hz}(\approx 50 \%$ load $)$ & $1.14 \%$ & $0 \%$ & $0 \%$ \\
\hline $63 / 20 \mathrm{~Hz}(\approx 75 \%$ load $)$ & $3.61 \%$ & $0.01 \%$ & $0 \%$ \\
\hline $84 / 20 \mathrm{~Hz}(\approx 99 \%$ load $)$ & $10.65 \%$ & $2.68 \%$ & $0.96 \%$ \\
\hline
\end{tabular}

PLI UNDER VARYING LOAD WITH PERFECT DECODING CAPABILITIES.

The SI size acts as a collision domain, resulting in more localized collisions with a smaller SI size and spread over the entire frame with an SI size of $100 \%$. In Figure 4 this effect is visualized by plotting the slots in which a collision occurred during one simulation run for an SI size of $20 \%$ and $100 \%$, respectively.

Third, the difference between the minimum and maximum PLI values, representing the effect of different network entry durations, is overall rather small and less decisive, compared to the other considered parameters.

3) Comparison to CSMA: For the next evaluation, considering random reservation durations, we limited the parameters of STDMA to an SI size of either $20 \%$ or $40 \%$ and a minimum CS of just 1 slot. These values are in accordance with the results shown in Table III and influenced by the fact that lower SI sizes contribute to more predictable inter transmission times. Table IV states the resulting PLI for this parametrization of STDMA and for CSMA with the settings stated in Table II. The PLI values of CSMA are increasing from about $1 \%$ with $50 \%$ load, to $3.6 \%$ with $75 \%$ load to $10.6 \%$ with $99 \%$ load. STDMA on the other hand is perfectly able to coordinate the channel with a load of $50 \%$. With a load of $75 \%$ and an SI size of $20 \%$, it occasionally happens that no free slot within the SI is available. With $99 \%$ load and perfect decoding capabilities the PLI for STDMA still stays rather low with better overall results for an SI size of $40 \%$. Further investigations with scenarios consisting of 168 vehicles transmitting with $10 \mathrm{~Hz}$ and 336 vehicles transmitting with $5 \mathrm{~Hz}$ (resulting in the same network load of $99 \%$ ) depict a slightly better PLI of $\approx 1 \%$ with an SI of $20 \%$. This effect can be explained by an increased SI interval size due to the reduced transmission rate.

4) Influence of starting-up behavior: When starting up several nodes per second, we observe occasional collisions due to two stations randomly selecting the identical slot for network entry. The probability hereby increases with the network load, as less free slots are available for stations to 


\begin{tabular}{l|c|c|c}
\hline \multirow{2}{*}{ \#nodes/rate } & CSMA & \multicolumn{2}{|c}{ STDMA } \\
\hline $42 / 20 \mathrm{~Hz}(\approx 50 \%$ load $)$ & $1.40 \%$ & $6.16 \%$ & $6.36 \%$ \\
\hline $84 / 10 \mathrm{~Hz}(\approx 50 \%$ load $)$ & $1.51 \%$ & $6.2 \%$ & $6.48 \%$ \\
\hline $63 / 20 \mathrm{~Hz}(\approx 75 \%$ load $)$ & $4.01 \%$ & $10.99 \%$ & $11.27 \%$ \\
\hline $84 / 20 \mathrm{~Hz}(\approx 99 \%$ load $)$ & $10.42 \%$ & $18.81 \%$ & $18.39 \%$ \\
\hline
\end{tabular}

TABLE V

PLI UNDER VARYING LOAD WITH NON PERFECT DECODING CAPABILITIES.

choose from for their network entry. However, the resulting PLI for every simulation carried out did not differ noticeably.

\section{Results for non-perfect decoding capabilities}

In this section we change the communication model to challenge the protocols (in particular, STDMA) with nondecodable but still detectable packets. For CSMA this does not introduce any new burden, since it is still able to sense the channel as busy over the entire simulation scenario strip with a length of $500 \mathrm{~m}$. However, STDMA can only decode and consequently extract reservation information from nodes positioned within a range of $300 \mathrm{~m}$. All other nodes still contribute to load on the channel and occupy slots that are sensed as busy. The resulting PLI values over the same parameter space considered before can be found in Table V.

As expected, PLI values for CSMA are not significantly different to the ones observed with perfect decoding. For STDMA the situation changes drastically as even with a load of just $50 \%$ the PLI increases to $\approx 6 \%$. The reason for this is twofold: First, reservation announcements of nodes further away than 300 meters cannot be 'understood' and, second, re-reservations are not understood either, which can have the effect that a slot is still considered as busy for one additional frame, even though the node switched to another slot and the previous slot is now free. Collisions resulting from non-perfect view on the vicinity are, thus, inevitable. With increasing load the PLI increases up to $\approx 18.5 \%$, whereby no clear difference between an SI size of $20 \%$ or $40 \%$ is observable.

Besides the PLI value itself, we also investigated the locations and the distance of the nodes that interfered with each other, respectively. Figure 5 visualizes the PLI for varying ranges, i.e. we evaluate the PLI if only incoordination of nodes within a certain distance are considered. It is clearly visible that up to a distance of 300 meters, which corresponds to the decoding range in our scenario, STDMA is still able to coordinate the nodes better than CSMA. The incoordination then likewise significantly increases, as the nodes are no longer able to extract reservation information.

\section{Summary And Outlook}

In this work, we have performed an in-depth investigation of STDMA, which recently gained interest as an alternative to CSMA in inter-vehicle communication networks. In contrast to CSMA, STDMA makes use of a distributed slot reservation mechanism to provide access to the wireless channel. All protocol elements have been explained in detail, analyzed and evaluated (by means of wireless network simulation) w.r.t. their ability to avoid slot allocation collisions. As our results

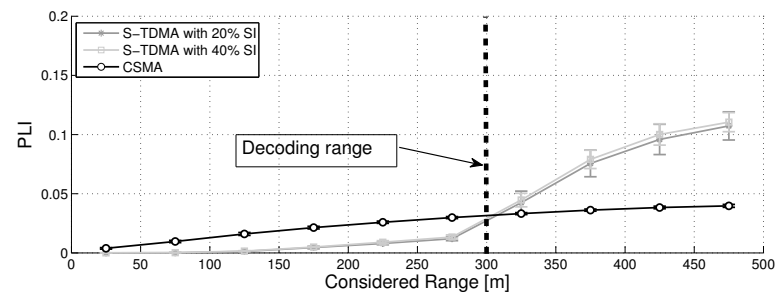

Fig. 5. Packet level incoordination w.r.t considered range of a node for the 63 nodes $20 \mathrm{~Hz}$ scenario with non-perfect decoding capabilities.

show, STDMA is a highly competitive alternative to CSMA in a periodic broadcast setting, and is able to avoid slot allocation collisions as long as all transmitted packets are successfully received by neighboring stations - even if the cumulative load offered by all stations is high. The introduction of packet reception errors reduces the effectiveness of STDMA significantly and its average performance drops below the one observed with CSMA in the same setup. This is intuitively expected as CSMA requires mostly physical carrier sensing to operate and does not primarily depend on the successful reception of packets and their contained control information.

While our results indicate the main characteristics of the analyzed protocols, a conclusive report on which protocols performs better requires further investigation. Therefore, we plan to extend the currently transmitter-based evaluation and to include receiver-based metrics, such as the packet reception probability or packet inter-arrival time distribution. We also plan to address advanced receivers and node mobility.

\section{REFERENCES}

[1] A. Alonso and C. Mecklenbräuker. Stabilization Time Comparison of CSMA and Self-Organizing TDMA for different Channel Loads in VANETS. In 12th Int'l Conf. on ITS Telecomm., pages 300-305, 2012.

[2] G. Bianchi, L. Fratta, and M. Oliveri. Performance Evaluation and Enhancement of the CSMA/CA MAC Protocol for 802.11 Wireless LANs. In 7th IEEE Int'l Symposium on Personal, Indoor and Mobile Radio Communications, volume 2, pages 392-396 vol.2, 1996.

[3] ETSI. Intelligent Transport Systems (ITS); STDMA recommended Parameters and Settings for Cooperative ITS; Access Layer Part. Technical report, European Telecommunications Standards Institute, 2012

[4] Eurocontrol. VHF Digital Mode 4. http://www.eurocontrol.int/services/ vhf-digital-mode- 4 .

[5] J. Grönkvist, J. Nilsson, and D. Yuan. Throughput of Optimal Spatial Reuse TDMA for Wireless Ad-hoc Networks. In 59th IEEE Vehicular Technology Conference, volume 4, pages 2156-2160. IEEE, 2004.

[6] A. C. V. Gummalla and J. O. Limb. Wireless Medium Access Control Protocols. IEEE Communications Surveys Tutorials, 3(2):2-15, 2000.

[7] ITU. Technical Characteristics for an Automatic Identification System Using Time Division Multiple Access in the VHF Maritime Mobile Band. Technical Report ITU-R M.1371-4, 2010.

[8] K. Bilstrup, E. Uhlemann, E.G. Ström, and U. Bilstrup. On the Ability of the 802.11p MAC Method and STDMA to Support RealTime Vehicle-to-Vehicle Communication. EURASIP Journal on Wireless Communications and Networking, 2008.

[9] K. Sjöberg, E. Uhlemann ,and E.G. Ström. How Severe is the Hidden Terminal Problem in VANETs when using CSMA and STDMA? In IEEE Vehicular Technology Conference, pages 1-5. IEEE, 2011.

[10] R. Kjellberg. Capacity and Throughput Using a Self Organized Time Division Multiple Access VHF Data Link in Surveillance Applications. Department of Computer and System Sciences, page 53, 1998.

[11] H. Lans. Position Indicating System. US Patent 5506587, April 1996.

[12] J. Mittag. Characterization, Avoidance and Repair of Packet Collisions in Inter-Vehicle Communication Networks. PhD thesis, Karlsruhe Institute of Technology, 2012. Available online via https://dsn.tm.kit.edu/ article.php?publication_id=248. 